\title{
Preface to special issue: behavioural types
}

\author{
SIMON J GAY G $^{\dagger}$ and ANTÓNIO RAVARA \\ ${ }^{\dagger}$ School of Computing Science, University of Glasgow, United Kingdom \\ ${ }^{\ddagger}$ Departamento de Informática, Universidade Nova de Lisboa, Portugal
}

Received 14 February 2014

Dedicated to the memory of Kohei Honda.

This is the first part of a two-part special issue on Behavioural Types, which has its origin in a workshop we organized in April 2011, in Lisbon. The aim of the workshop was to bring together the active and expanding community of researchers using type-theoretic approaches to describe and analyse behavioural aspects of software. A particular concern of this field is the identification and description of structured communication in concurrent and distributed systems, but behavioural typing also addresses issues of liveness, fairness, deadlock-freedom, security, observable equivalence and typestate.

The call for papers attracted an impressive number of submissions, which were each reviewed by three referees in order to make a selection for the special issue. After a further round of reviewing of revised versions of the papers, the final result is a collection of nine excellent articles spread across two issues.

In December 2012, our community was shocked by the unexpected death of Kohei Honda. Kohei was the originator of session types, which are a central part of the field of behavioural types, and was one of the most active, influential and inspiring researchers in the field. He is a co-author of one of the papers in this special issue, and his wife and collaborator, Nobuko Yoshida, is a co-author of that paper and two others. We dedicate this special issue to Kohei's memory.

Papers in this issue

- Global Escape in Multiparty Sessions, by Sara Capecchi, Elena Giachino and Nobuko Yoshida, extends the established theory of multiparty session types by adding an exception mechanism to allow the participants in a protocol to collaboratively recover from an unexpected situation.

- Linearity, Session Types and the Pi Calculus, by Marco Giunti and Vasco Vasconcelos, re-examines the foundations of binary session types in pi calculus and defines a type system which is more flexible than the previous standard approaches.

- Global Progress for Dynamically Interleaved Multiparty Sessions, by Mario Coppo, Mariangiola Dezani, Nobuko Yoshida and Luca Padovani, considers the question of progress in multiparty sessions. It extends previous work by establishing progress properties without requiring a restriction that sessions on different channels are not interleaved.

- On Asynchronous Eventful Session Semantics, by Dimitrios Kouzapas, Nobuko Yoshida, Raymond $\mathrm{Hu}$ and Kohei Honda, applies session types to event-driven programming 
and uses a theory of behavioural equivalence to prove correctness of a transformation from multithreaded to event-driven programs.

The work presented in these special issues covers research goals being actively pursued by the community. COST Action IC1201: Behavioural Types for Reliable Large-Scale Software Systems (BETTY) (http://www.behavioural-types.eu) provides coordination for European research in this field. The initial workshop has been followed by the BEAT workshop series (http://beat2.behavioural-types.eu) to provide a forum for continuing collaborative research on behavioural types.

Guest editors of the special issue:

Simon Gay

António Ravara 